\section{Vagus nerve stimulation and refractory depression}

\author{
Please can you switch me on doctor?
}

KEITH MATTHEWS and MUFTAH S. ELJAMEL

As highlighted in the recent systematic review by Stimpson et al (2002), we know little about the relative efficacies of the treatment interventions that are routinely deployed for those with depressive disorders that have not responded to standard, first-line treatment approaches. Accepted practice is, at best, systematic 'trial and error', with sequential antidepressant monotherapy and/or combinations of antidepressant drugs with 'augmenting agents'. This haphazard medicinal approach to managing the patient with poorly responsive depressive disorder is compounded by the lack of available information on the effectiveness and optimal role for psychological treatment methods. Given the high prevalence of depressive disorders and their frequent presentation to both primary and secondary care health services, it is a source of embarrassment that we remain so ignorant. Nevertheless, it is against this backdrop of uncertainty that we must now evaluate the relative merits of a novel intervention that has captured considerable media and lay interest: vagus nerve stimulation $\left(\mathrm{VNS}^{\mathrm{TM}}\right)$.

\section{WHAT IS VNSTM AND HOW WAS IT DEVELOPED?}

Vagus nerve stimulation has come to mean the electrical stimulation of the cervical portion of the left vagus nerve and is usually achieved using a specific commercial electronic device - the NeuroCybernetic Prosthesis $\left(\mathrm{NCP}^{\circledR}\right)$ system, manufactured by Cyberonics Inc., (Houston, TX, USA). During clinical trials investigating the potential of $\mathrm{VNS}^{\mathrm{TM}}$ as a treatment for medication-refractory epilepsy, dramatic changes in mood were noted in some participants. These changes appeared unrelated to the presence or absence of beneficial effects on seizure activity. With an implantation registry of over 16000 patients, the $\mathrm{NCP}^{\circledR}$ system has been demonstrated to be a safe and effective intervention in refractory epilepsy (Schachter, 2002). In many respects, use of the $\mathrm{NCP}^{\circledR}$ system is akin to that of an implanted cardiac pacemaker. A bipolar the left vagus nerve and connected subcutaneously to an implantable, programmable pulse generator located under the skin of the anterior left chest wall. Stimulation of the right vagus nerve is avoided because of its potential effects on cardiac function. The pulse generator can be programmed by telemetry using a wand, with control software running on a standard personal computer. With this system, it is possible to program the device in the clinic, to retrieve data and to perform simple diagnostic procedures simply by placing the wand over the anterior chest wall. Each patient is issued with a small, powerful magnet that can be used either to trigger the generator or to suspend its operations. The stimulus triggering facility for depression. Its value lies in offering the capacity to trigger stimulation during an developing seizure. The currents applied to the vagus nerve are of milliampere magnitude (standard range 1.0-2.5), with a pulse width of around $500 \mu$ s and stimulation frequencies of $20-30 \mathrm{~Hz}$. With these standard settings (stimulator on for $30 \mathrm{~s}$ every $5 \mathrm{~min}$ ), predicted battery life is between 5 and 10 years.

\section{WHAT IS THE VAGUS NERVE AND WHAT DOES IT DO?}

The vagus is the tenth cranial nerve and is best known as a conduit for the parasympathetic outflow regulating autonomic functions in the thorax and abdomen (e.g. modulating heart rate and gastric acid stimulating electrode is wrapped around is not considered appropriate with $\mathrm{VNS}^{\mathrm{TM}}$ epileptic aura in an attempt to abort a secretion). Its name originates from the Latin verb vagor, meaning 'to rove' or to 'wander', and is a reflection of its rambling, tortuous course through the neck, thorax and abdomen. Both left and right vagus nerves are composed of efferent and afferent fibres with a mixture of sensory and motor functions. The largest component of the left vagus is the afferent sensory input, which constitutes around $80 \%$ of the total fibre content. These fibres carry information relevant to the perception of hunger, satiety and pain. The same fibres are also assumed to mediate the therapeutic effects of $\mathrm{VNS}^{\mathrm{TM}}$. Afferent sensory fibres relay information from extracranial sources to the nodose ganglion and the nucleus of the tractus solitarius in the medulla. In turn, these structures project to the brainstem median raphe nucleus and locus coereuleus, the dominant sources of serotonergic and noradrenergic innervation, respectively, of limbic and neocortical structures. Hence, direct electrical stimulation of the extracranial vagus nerve can have an impact on the activity of major brain-stem structures. In turn, these have the capacity to modulate the activity of cortical and limbic structures that are considered relevant to depression (e.g. anterior cingulate and orbitofrontal cortices, hippocampus and amygdala). Thus, VNS ${ }^{\mathrm{TM}}$ offers the potential to modify brain activity by means of electrical stimulation without intracranial surgery or the emotive convulsive effect that diminishes the acceptability of electroconvulsive therapy (ECT).

\section{WHAT IS KNOWN ABOUT THE EFFECTS AND ADVERSE EFFECTS OF VNS ${ }^{\text {TM? }}$}

\section{Effects of VNS ${ }^{\text {TM }}$}

As with many putative antidepressant treatments, VNS ${ }^{\mathrm{TM}}$ results in a complex cascade of central neurochemical changes, and the identification of a convincing mechanism of antidepressant action remains elusive. Indeed, most available information relates to the use of $\mathrm{VNS}^{\mathrm{TM}}$ in experimental animals and in patients with epilepsy. At the most simplistic level, VNS ${ }^{\mathrm{TM}}$ appears to exert substantial effects on crude indices of noradrenergic, serotonergic and glutaminergic systems. Similarly, positron emission tomography studies conducted in patients with epilepsy suggest that $\mathrm{VNS}^{\mathrm{TM}}$ increases blood flow in the medulla, 
thalamus, hypothalamus, insula and postcentral gyrus, particularly on the contralateral side (Henry et al, 1998). There are also bilateral reductions in blood flow in limbic cortical structures, the cingulate gyri, amygdala and hippocampus. Whether such changes can be observed in depressed patients remains to be determined.

\section{Adverse effects of VNS ${ }^{\text {TM }}$}

Extensive experience with $\mathrm{VNS}^{\mathrm{TM}}$ for epilepsy suggests that adverse effects can be considered under two main groupings: those directly related to the performance of surgery and those directly related to stimulation itself. The consequences of nerve damage and the range of stimulationrelated adverse effects can be predicted by knowledge of the anatomy and functions of the vagus nerve. There is a small risk of implant-related infection (around 1\%), with a similarly low risk of physical damage to the vagus nerve. The risk of nerve damage can be reduced if the stimulator is left inactive for 10-14 days after implantation. Around one-third of patients experience significant pain around the implant wound. Discomfort tends to recede with healing. The commonest stimulationrelated adverse effects are headache, neck, throat, pharynx, jaw and dental pain, hoarseness, alteration or loss of voice, cough and difficulty in swallowing (Sackeim et al, 2001). However, these experiences appear to be confined to periods during which the stimulator is active and generally can be ameliorated or abolished by reducing the intensity of the stimulation current. There is a general increase in tolerability of stimulation with the passage of time but, at least in our experience, patients occasionally will describe an unexplained worsening of stimulation-related discomfort. It is rare for patients to have to use the magnet to discontinue stimulation but many find reassurance in knowing that the facility is available. Some patients (usually male) may describe stimulation settings as tolerable despite their obviously aversive quality. It is sometimes necessary to allow patients to test out the tolerability of stimulation settings for a period of hours before they depart. Asking patients to drink a glass of water during a period of stimulation can provide useful confirmation that swallowing is not unduly affected.

\section{HOW EFFECTIVE IS VNSTM IN TREATMENT-REFRACTORY DEPRESSION?}

\section{Acute efficacy}

In two open pilot studies, 60 patients with chronic, treatment-refractory major depressive episodes were recruited and implanted with the $\mathrm{NCP}^{\circledR}$ system $(10$ weeks of $\mathrm{VNS}^{\mathrm{TM}}: n=30$, Rush et al, 2000; $n=30$ plus a further 30, Sackeim et al, 2001). Combining the two data-sets, 18 participants met the a priori criteria for 'clinical response' within 12 weeks: $\geqslant 50 \%$ reduction in score on the 28-item version of the Hamilton Rating Scale for Depression (HRSD; Hamilton, 1960). One participant responded during the 2-week, single-blind, post-implantation period when the stimulator was inactive. Hence, after 10 weeks of active stimulation $18 / 59$ participants $(31 \%)$ were judged to have responded. Of these, nine $(15 \%)$ met the criteria for 'complete response': a score of $\geqslant 10$ on the HRSD.

Evaluation of outcome according to other measures, including self-report, revealed broadly similar response rates. Three participants worsened during this period of treatment. Adverse effect rates and the profile of events were broadly similar to those reported in epilepsy $\mathrm{VNS}^{\mathrm{TM}}$ studies. No participants ceased stimulation during this phase as a result of adverse effects. The commonest adverse effects were cough, hoarseness and voice alteration during stimulation. Wound discomfort was reported by around one-third of participants. There were, however, two isolated clinical events that are of potential significance within such a clinical population if $\mathrm{VNS}^{\mathrm{TM}}$ were to become a commonly used treatment. Two participants developed pathological mood elevation, although one was the placebo responder. A further participant with multiple cardiac risk factors experienced myocardial infarction within $48 \mathrm{~h}$ of starting VNS ${ }^{\mathrm{TM}}$. This, however, did not lead to withdrawal from the study and detailed monitoring of cardiac physiology during the early stages of $\mathrm{VNS}^{\mathrm{TM}}$ did not suggest increased risk.

\section{Longer-term efficacy}

Data on longer-term efficacy are, regrettably, sparse. Of those who responded to acute VNS ${ }^{\mathrm{TM}}$, benefit appears to have been maintained at 12 months according to a naturalistic follow-up study, with a further
9 months of $\mathrm{VNS}^{\mathrm{TM}}$ (Marangell et al, 2002). Data obtained following 24 months of $\mathrm{VNS}^{\mathrm{TM}}$ have been presented at international conferences such as the 2001 US Psychiatric and Mental Health Congress, but as yet remain unpublished. Initial impressions are favourable, with low rates of withdrawal from treatment and an apparent enhancement of efficacy with prolonged exposure to $\mathrm{VNS}^{\mathrm{TM}}$. Interestingly, there was no apparent loss of efficacy with increasing duration of $\mathrm{VNS}^{\mathrm{TM}}$ - a phenomenon that, if reliable, would represent a unique property for any therapy for depression. Indeed, some participants who had not responded during the acute study subsequently met the criteria for response at $\mathbf{1 2}$ months, suggesting that it may be necessary to persist with treatment for at least a year before concluding that it is ineffective. These data are consistent with those derived from longer-term epilepsy VNS ${ }^{\mathrm{TM}}$ studies where benefit appears to accrue with persistence of stimulation (Schachter, 2002).

\section{WHAT IS THE PLACE OF VNSTM IN THE MANAGEMENT OF REFR ACTORY DEPRESSION?}

If any treatment for chronic, refractory depressive disorder were to offer the prospect of sustained, clinically significant change in $20-30 \%$ of patients, this would represent a major therapeutic advance. However, history tells us that psychiatric interventions that appear highly promising in open studies rarely impress when subjected to the rigours of the randomised controlled trial. Indeed, a favourable acute response rate of one-third was reported by Moniz in his open series of alcohol injection and blade leucotomies performed on patients with depression, catatonia and/or schizophrenia during the 1930s (Moniz, 1936). Data from controlled evaluations of $\mathrm{VNS}^{\mathrm{TM}}$ for depression are, unfortunately, still awaited. A randomised controlled trial has been attempted in the USA but errors of conduct have rendered the data uninterpretable. A significant proportion of participants randomised to the 'active treatment' limb of this unpublished study did not have stimulator settings increased and optimised as mandated by protocol. As a consequence, both treatments performed equally well. A further impediment has been the difficulty in constructing an appropriate control condition 
because, for most individuals, $\mathrm{VNS}^{\mathrm{TM}}$ elicits distinctive somatic sensations during stimulus application. If such difficulties can be overcome, large-scale, multi-centre, controlled evaluations will be required before $\mathrm{VNS}^{\mathrm{TM}}$ can be recommended with confidence.

A preliminary analysis of differences between responders and non-responders on the open study (Sackeim et al, 2001) suggested that the probability of $\mathrm{VNS}^{\mathrm{TM}}$ response diminished with increasing degree of treatment resistance - both in terms of the range and vigour of antidepressant medication trials and previous failure to respond to ECT. When the outcomes from the acute study were first published, our unit was immediately interested in evaluating the relative merits of $\mathrm{VNS}^{\mathrm{TM}}$ compared with ablative neurosurgery. Despite obtaining ethical permission to conduct a randomised comparison of $\mathrm{VNS}^{\mathrm{TM}}$ with ablative anterior cingulotomy, we have been unable to proceed. Each patient presenting to our service having fulfilled the stringent criteria for treatment adequacy required before proceeding to neurosurgery for mental disorder (Matthews \& Eljamel, 2003) has expressed a strong preference for one treatment over the other-not always in favour of $\mathrm{VNS}^{\mathrm{TM}}$.

\section{CONCLUSIONS}

Vagus nerve stimulation may represent a major advance in the management of

KEITH MATTHEWS, MD, PhD, MUFTAH S. ELJAMEL, MD, Departments of Psychiatry and Surgical Neurology, University of Dundee, Dundee, UK

Correspondence: Keith Matthews, Department of Psychiatry, University of Dundee, Ninewells Hospital and Medical School, Dundee DDI 9SY, UK. Tel: 01382 632121; fax: 01382633923 ;

e-mail:k.matthews@dundee.ac.uk

(First received 31 January 2003, final revision 3 April 2003, accepted 7 April 2003)

chronic and treatment-refractory depressive disorders. Ultimately, it may offer greatest benefit if employed in the management of relatively less chronic, refractory and disabled patients. However, definitive clinical trials are awaited and it must still be considered an 'experimental' treatment. Provision of firm guidance concerning its likely place in the management of depression is hindered by our poor understanding of the relative efficacy of other medicinal and psychological interventions.

\section{DECLARATION OF INTEREST}

None.

\section{REFERENCES}

Hamilton, M. (1960) A rating scale for depression. Journal of Neurology, Neurosurgery and Psychiatry, 23, 56-62.

Henry, T. R., Bakay, R. A., Votaw, J. R., et al (1998) Brain blood flow alterations induced by therapeutic vagus nerve stimulation in partial epilepsy. I. Acute effects at high and low levels of stimulation. Epilepsia, 39 983-990.
Marangell, L. B., Rush, A. J., George, M. S., et al (2002) Vagus nerve stimulation (VNS) for major depressive episodes: one year outcomes. Biological Psychiatry, 5I, 280-287.

Matthews, K. \& Eljamel, M. S. (2003) Status of neurosurgery for mental disorder in Scotland. Selective literature review and overview of current clinical activity. British Journal of Psychiatry, 182, 404-4II.

Moniz, E. (1936) Tentatives Opératoires dans le Traitement de Certaines Psychoses. Paris: Masson.

Rush, A. J., George, M. S., Sackeim, H. A., et al (2000) Vagus nerve stimulation (VNS) for treatment resistant depressions: a multicenter study. Biological Psychiatry, 47, 276-286.

Sackeim, H. A., Rush, A. J., George, M. S., et al (200I) Vagus nerve stimulation (VNS) for treatmentresistant depression: efficacy, side effects and predictors of outcome. Neuropsychopharmacology, 25, 713-728.

Schachter, S. C. (2002) Vagus nerve stimulation therapy summary: five years after FDA approval. Neurology, 59 (suppl. 4), SI5-S20.

Stimpson, N., Agrawal, N. \& Lewis, G. (2002) Randomised controlled trials investigating pharmacological and psychological interventions for treatment-refractory depression. Systematic review. British Journal of Psychiatry, 18I, 284-294. 